1998-6

\title{
Fuel Equalized Retargeting for Separated Spacecraft Interferometry
}

Timothy McLain

Mechanical Engineering Department, Brigham Young University, mclain@byu.edu

Randal W. Beard

Department of Electrical and Computer Engineering, Brigham Young University, beard@ee.byu.edu

Fred Y. Hadaegh

Jet Propulsion Laboratory, California Institute of Technology

Follow this and additional works at: https://scholarsarchive.byu.edu/facpub

Part of the Mechanical Engineering Commons

\section{Original Publication Citation}

Beard, R. and McLain, T. Fuel Equalized Retargeting for Separated Spacecraft Interferometry, Proceedings of the American Control Conference, vol. 3, pp. 1580-1584, June 1998, Philadelphia, Pennsylvania.

\section{BYU ScholarsArchive Citation}

McLain, Timothy; Beard, Randal W.; and Hadaegh, Fred Y., "Fuel Equalized Retargeting for Separated Spacecraft Interferometry" (1998). Faculty Publications. 1940.

https://scholarsarchive.byu.edu/facpub/1940

This Conference Paper is brought to you for free and open access by BYU ScholarsArchive. It has been accepted for inclusion in Faculty Publications by an authorized administrator of BYU ScholarsArchive. For more information, please contact ellen_amatangelo@byu.edu. 


\section{Fuel Equalized Retargeting for Separated Spacecraft Interferometry}

\author{
Randal W. Beard \\ Electrical Eng. Dept. \\ Brigham Young University \\ beard@ee.byu.edu
}

\author{
Timothy W. McLain \\ Mechanical Eng. Dept. \\ Brigham Young University \\ tmclain@et.byu.edu
}

\author{
Fred Y. Hadaegh \\ Jet Propulsion Laboratory \\ California Institute of Technology \\ fred.y.hadaegh@jpl.nasa.gov
}

\begin{abstract}
Motivated by NASA's proposed Deep Space 3 interferometer mission, this paper considers the problem of reorienting a constellation of spacecraft such that the total fuel distributed across the constellation is both conserved and expended uniformly. The spacecraft constellation is controlled to reorient as if it were a rigid body. Two approaches to fuel equalization are investigated. The first approach picks a point of rotation a priori that optimizes an objective function that trades off minimum-fuel maneuvers and maneuvers that equalize the fuel. Since the point of rotation is selected a priori and is fixed during the rotation, this approach is open-loop in that it cannot adjust to unpredicted, or inaccurately modeled fuel use. The second approach is closed-loop in that the point of rotation is caused to have second-order dynamics that track the center of unavailable fuel mass. Intuitively, the center of fuel mass will dynamically change to be close to spacecraft that are low on fuel. Simulation results for a four-spacecraft constellation restricted to a plane are given.
\end{abstract}

\section{Introduction}

This paper considers the problem of retargeting a constellation of spacecraft flying in formation. The problem is motivated by the objective of retargeting spaced-based interferometers that are configured from free-flying separated spacecraft. NASA's proposed Deep Space 3 interferometer measures distances between spacecraft with a laser metrology system. Initializing this system is a timely and complicated process. Therefore, when the constellation is reoriented to point at another target, the relative distance and orientation between the spacecraft need to be maintained within a specified tolerance. Consequently, the constellation needs to be reoriented as a virtual rigid body. To retarget a constellation, individual spacecraft may be required to use different amounts of fuel. After several reorientations, some of the spacecraft may have used all of their fuel while others may have expended very little. The objective of this paper is to derive two constellation retargeting algorithms that ensure that fuel consumption is equalized among the spacecraft.

Our basic approach is twofold. The first step incorporates both the kinematic constraints imposed on the spacecraft by the constellation, and the maximum thrust capability of each spacecraft, to derive position and velocity trajectories for each spacecraft. The second step is to use PD controllers to track these trajectories.

Of paramount importance is the point about which the constellation is reoriented. If the spacecraft are located an equal distance from the point of rotation, then the fuel expended by each spacecraft during a reorientation will be equal. This paper will explore two approaches to picking the point of rotation. The first approach is to a priori pick a point of rotation such that the fuel distribution at the end of the maneuver is equalized and the total fuel expended by the constellation is as small as possible. This constitutes an open-loop approach to picking the point of rotation. Once the point of rotation is selected, it is fixed and cannot adapt to reflect fuel expenditure that may be different than what was predicted a priori. This approach will be discussed in Section 3 . The second approach is to dynamically change the point of reorientation according to the amount of fuel possessed by each spacecraft. The idea is to move the point of rotation so that it is close to spacecraft that are low on fuel. This approach will be discussed in Section 4 .

Formation flying for separated spacecraft is a relatively new area of research. The problem of maintaining a fixed formation of spacecraft has been considered in [1]. Continuous rotation of a constellation about a fixed axis has been addressed in [2]. Initializing a formation of spacecraft has been considered in [3]. To our knowledge, this paper is the first time that the problem of fuel equalization has been considered.

\section{Model and Assumptions}

For simplicity of presentation, we will restrict our attention to rotations in a plane. The planar case contains 
all of the essential features of fuel equalization and the extension to three dimensions does not require any new ideas. Also, since the primary drain on the fuel will be translational motion of the spacecraft, the rotational dynamics of the spacecraft will be ignored. Assuming that the fuel expended by a spacecraft is proportional to the norm of the applied thrust, the model for each spacecraft is

$$
\begin{aligned}
M_{i} \ddot{r}_{i} & = \begin{cases}\operatorname{sat}_{\alpha_{i}}\left(u_{i}\right) ; & f_{i}(t)>0 \\
0 ; & \text { otherwise }\end{cases} \\
\dot{f}_{i} & = \begin{cases}-\gamma\left\|\operatorname{sat}_{\alpha_{i}}\left(u_{i}\right)\right\| ; & f_{i}(t)>0 \\
0 ; & \text { otherwise }\end{cases}
\end{aligned}
$$

where $M_{i}$ is the mass of the spacecraft, $r_{i} \in \mathbb{R}^{2}$ is its position in the inertial frame, $u_{i} \in \mathbb{R}^{2}$ is the control thrust, $f_{i}$ is the amount of fuel in the tank of the $i^{\text {th }}$ spacecraft, $\gamma$ is a proportionality constant and sat $\alpha_{i}$ is the saturation function with limits $\pm \alpha_{i}$. The constellation is assumed to be in free space.

The coordinate frame geometry is shown in Figure 1. The fixed inertial frame is $O, C$ is the constellation frame

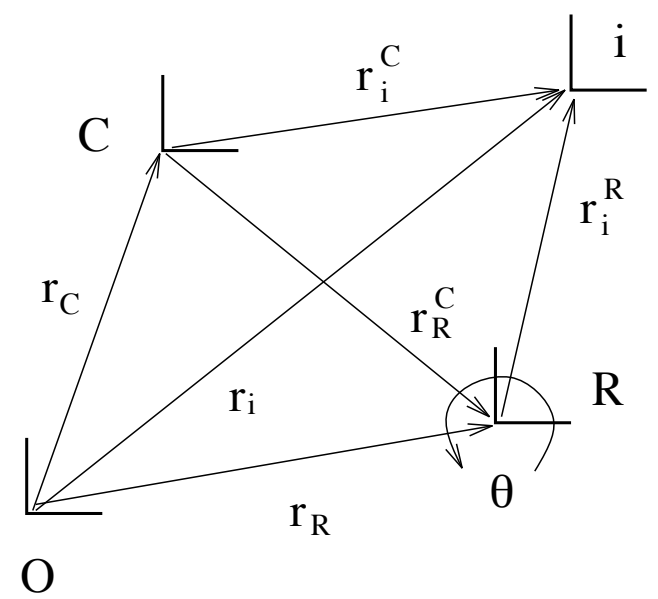

Figure 1: Coordinate frames.

(i.e. $r_{i}^{C}$ is fixed and $v_{i}^{C}=0$ where $v=\dot{r}$ is the velocity), $R$ is the rotation frame and $i$ is the frame of the $i^{\text {th }}$ spacecraft in the constellation. The angle of rotation of the constellation about $R$ is denoted by $\theta$.

\section{Fixed Point of Rotation}

In this section we derive an algorithm for picking the location of the rotation point, i.e. $r_{R}$, such that given the initial fuel distribution $\left\{f_{1}(0), \ldots, f_{N}(0)\right\}$, the final fuel distribution $\left\{f_{1}\left(t_{f}\right), \ldots, f_{N}\left(t_{f}\right)\right\}$ minimizes the following functional:

$$
\begin{aligned}
J=\min _{r_{R}}\{ & \sum_{i=1}^{N}\left(f_{i}(0)-f_{i}\left(t_{f}\right)\right)^{2} \\
& \left.+\mu \sum_{i=1}^{N} \frac{f_{i}\left(t_{f}\right)}{\sum_{j=1}^{N} f_{j}\left(t_{f}\right)} \log \frac{f_{i}\left(t_{f}\right)}{\sum_{j=1}^{N} f_{j}\left(t_{f}\right)}\right\} .
\end{aligned}
$$

The first term in this functional represents the total amount of fuel expended by the constellation. The second term is motivated by the negative entropy of a probability distribution [4], which is minimum for a uniform distribution, i.e., the second term will be minimized when $f_{i}\left(t_{f}\right)=f_{j}\left(t_{f}\right)$ for all $i, j \in\{1, \ldots, N\}$. To minimize this functional, we need to express $f_{i}\left(t_{f}\right)$ in terms of $r_{R}$.

Suppose that the constellation is to be rotated about $R$ by an amount $\hat{\theta}$. Each spacecraft is constrained to rotate at the same angular rate about $R$. Let $\ddot{\phi}=$ $\sqrt{\ddot{\theta}^{2}+\dot{\theta}^{4}}$ denote the generalized (combination of tangential and centripetal) acceleration of the spacecraft. When $i^{\text {th }}$ spacecraft is thrusting, its equation of motion can be written as $M_{i}\left\|r_{i}^{R}\right\| \ddot{\phi}=\alpha_{i}$. Since $\ddot{\phi}$ is identical for each spacecraft, we have that

$$
\frac{\alpha_{1}}{M_{1}\left\|r_{1}^{R}\right\|}=\cdots=\frac{\alpha_{N}}{M_{N}\left\|r_{N}^{R}\right\|}
$$

Since the maximum angular acceleration of the constellation will be determined by the thrust limit of the spacecraft furthest from $R$, and since $r_{i}^{R}=r_{i}(0)-r_{R}$, if

$$
\beta=\arg \max _{1 \leq i \leq N}\left\|r_{i}(0)-r_{R}\right\|
$$

then the maximum angular acceleration of the constellation is given by

$$
A \triangleq \frac{\alpha_{\beta}}{M_{\beta}\left\|r_{i}^{R}\right\|}=\frac{\alpha_{\beta}}{M_{\beta}\left\|r_{\beta}(0)-r_{R}\right\|} .
$$

If the constellation is rotating at its maximum angular acceleration then the thrust for each spacecraft is given by

$$
\alpha_{i}=\frac{\alpha_{\beta} M_{i}\left\|r_{i}(0)-r_{R}\right\|}{M_{\beta}\left\|r_{\beta}(0)-r_{R}\right\|}
$$

A trajectory for the constellation is found by constructing a minimum-fuel, minimum-time trajectory for the constellation. As shown in [5], a minimum-time, minimum-fuel trajectory for a double-integrator plant with actuator saturation is given by a bang-off-bang control trajectory. Therefore we obtain the following possi- 
ble trajectories for $\theta$, the angle of rotation about $R$ :

$$
\begin{aligned}
& \ddot{\theta}(t)= \begin{cases}A ; & 0 \leq t \leq t_{w} \\
0 ; & t_{w} \leq t \leq t_{f}-t_{w} \\
-A ; & t_{f}-t_{w} \leq t \leq t_{f},\end{cases} \\
& \dot{\theta}(t)= \begin{cases}A t ; & 0 \leq t \leq t_{w} \\
A t_{w} ; \quad t_{w} \leq t \leq t_{f}-t_{w} & \\
A\left(t_{f}-t\right) ; \quad t_{f}-t_{w} \leq t \leq t_{f},\end{cases} \\
& \theta(t)= \begin{cases}\frac{1}{2} A t^{2} ; & 0 \leq t \leq t_{w} \\
A t_{w} t-\frac{1}{2} A t_{w}^{2} ; & t_{w} \leq t \leq t_{f}-t_{w} \\
A t_{w}\left(\left(t_{f}-t_{w}\right)-\frac{1}{2} A\left(t_{f}-t\right)^{2} ;\right. & t_{f}-t_{w} \leq t \leq t_{f},\end{cases}
\end{aligned}
$$

where $t_{w}$ is the width of the thrust pulse. Using the fact that $\hat{\theta}=\theta\left(t_{f}\right)=A t_{w}\left(t_{f}-t_{w}\right)$ we get

$$
\begin{aligned}
& t_{f}=\frac{\hat{\theta}}{A t_{w}}+t_{w} \\
& t_{w}=\frac{t_{f}}{2}-\sqrt{\frac{t_{f}^{2}}{2}-\frac{\hat{\theta}}{A}} .
\end{aligned}
$$

Assuming that the profile for the magnitude of thrust for each spacecraft is given by

$$
\left\|u_{i}(t)\right\|= \begin{cases}\alpha_{i} ; & 0 \leq t \leq t_{w} \\ 0 ; & t_{w} \leq t \leq t_{f}-t_{w} \\ \alpha_{i} ; & t_{f}-t_{w} \leq t \leq t_{f}\end{cases}
$$

the amount of fuel consumed by the $i^{\text {th }}$ spacecraft during the maneuver is $f_{i}(0)-f_{i}\left(t_{f}\right)=2 \gamma t_{w} \alpha_{i}$ which implies that

$$
f_{i}\left(t_{f}\right)=f_{i}(0)-2 \gamma t_{w} \alpha_{i}
$$

To complete the formulation, we need to express $t_{w}$ in terms of $A$ and $\alpha_{i}$. Define the total amount of fuel used by the constellation during the maneuver to be

$$
F \triangleq \sum_{i=1}^{N}\left(f_{i}(0)-f_{i}\left(t_{f}\right)\right)=2 \gamma t_{w} \sum_{i=1}^{N} \alpha_{i} .
$$

Substituting from equation (7) and solving for $t_{f}$ we get

$$
t_{f}=\frac{F}{2 \gamma \sum \alpha_{i}}+\frac{2 \gamma \hat{\theta} \sum \alpha_{i}}{A F} .
$$

To trade off minimum-fuel and minimum-time we minimize the function

$$
\begin{aligned}
J_{2} & =\min \left(F+\lambda t_{f}\right) \\
& =\min _{F}\left\{F+\lambda\left[\frac{F}{2 \gamma \sum \alpha_{i}}+\frac{2 \gamma \hat{\theta} \sum \alpha_{i}}{A F}\right]\right\}
\end{aligned}
$$

for $\lambda>0$. The minimum-time, minimum-fuel tradeoff is now explicitly in terms of $\lambda$. For $\lambda=0$ we get the minimum-fuel (maximum-time, i.e. infinity) solution, as $\lambda \rightarrow \infty$ we get the minimum-time (maximum-fuel) solution. Setting the derivative of the above expression to zero and solving for $F$ gives

$$
F=2 \gamma \sum \alpha_{i} \sqrt{\frac{\lambda \hat{\theta}}{2 \gamma A \sum \alpha_{i}+A \lambda}} .
$$

Equations (10), (9) and (7) can be used in equation (8) to express $f_{i}\left(t_{f}\right)$ in terms of $A$ and $\alpha_{i}$. Equations (3), (4) and (5) are then used to express $f_{i}\left(t_{f}\right)$ in terms of $r_{R}$. We can now use a minimization software, like Matlab's fmins function to find $r_{R}$ to minimize the objective function given in equation (2). The minimization routine is initialized using the center of fuel mass at time $t=0$

$$
\frac{\sum_{i=1}^{N} r_{i}(0) / f_{i}(0)}{\sum_{j=1}^{N} 1 / f_{j}(0)}
$$

\section{Dynamic Point of Rotation}

In this section, we present a strategy for dynamically locating the point of rotation of a spacecraft constellation undergoing retargeting. The primary advantage of dynamically locating the rotation point of the constellation is that it provides a way to ensure uniform fuel usage between satellites in unanticipated situations, such as a spacecraft expending fuel at a greater rate than projected due to a thruster malfunction.

The strategy for dynamically locating the rotation point is similar in many respects to the fixed-point strategy with the obvious exception that the fuel distribution between satellites is monitored continually throughout the retargeting operation and the location of the rotation point $r_{R}$ is adjusted accordingly. The computation of individual spacecraft accelerations is significantly more complicated for the dynamic-point approach due to coriolis terms that are introduced by the motion of the rotation point. Spacecraft accelerations introduced by excessive motion of the rotation point can also result in inefficient fuel usage due to the additional thrust required to maintain rotation about the moving point.

As with the fixed-point strategy, the spacecraft positions are constrained in the constellation frame such that $r_{i}^{C}$ is fixed and $v_{i}^{C}=0$ and the desired rotation of the constellation $\theta$ is specified.

The first step in the dynamic-point strategy is to establish the rotation trajectory for the entire constellation, i.e. specifying $\theta(t), \dot{\theta}(t)$, and $\ddot{\theta}(t)$. We again assume that the constellation moves according to a bang-off-bang control trajectory. The maximum possible radius of rotation for any spacecraft at any time during retargeting is assumed to be the maximum distance between any two spacecraft in the constellation. For a given final time $t_{f}$, rotation angle $\hat{\theta}$, maximum radius of rotation $r_{\text {max }}^{R}$, 
and maximum acceleration capability for each spacecraft $a_{\max }$, the switching time $t_{w}$ of a bang-off-bang (minimum thrust pulse width) trajectory can be determined by the solution to the nonlinear equation

$$
a_{\max }-r_{\max }^{R} \sqrt{\left(\frac{\hat{\theta}}{t_{f}-t_{w}}\right)^{4}+\left(\frac{\hat{\theta}}{t_{w}\left(t_{f}-t_{w}\right)}\right)^{2}} .
$$

For simplicity, we assume that $a_{\max }=\alpha_{i} / M_{i}$ is the same for each spacecraft in the constellation. An alternative would be to let $a_{\max }$ be the maximum acceleration of the spacecraft with the lowest acceleration capability.

With the trajectory of the constellation template determined, the "initial conditions" of the retargeting maneuver must be specified. This includes the initial position of the rotation point $r_{R}(0)$, the initial velocity of the rotation point $v_{R}(0)$ (typically zero), and the initial position of the constellation reference point relative to the point of rotation $r_{R}^{C}$. Also included is the initial fuel mass of each spacecraft $f_{i}(0)$.

Once the initial configuration of the spacecraft constellation has been determined, the retargeting maneuver is initiated according to the rotation trajectory $\theta(t)$ determined previously. This represents the rotation of the constellation template with respect to the inertial reference frame.

The motion of the rotation point is determined by examining the available fuel mass of each of the spacecraft throughout the maneuver. The desired point of rotation is found by computing the center of unavailable fuel mass $r_{u f}:$

$$
r_{u f}(t)=\frac{\sum_{i=1}^{N} r_{i}(t) / f_{i}(t)}{\sum_{i=1}^{N} f_{i}(t)}
$$

As the retargeting motion is carried out, the center of unavailable fuel mass will move toward the spacecraft with the least amount of fuel. The center of unavailable fuel mass becomes the desired rotation point for the constellation $r_{R d}$.

The fact that the rotation point is free to move implies that the rotation point has an acceleration associated with it. The acceleration of the rotation point is required to compute the acceleration of each spacecraft, which enables the calculation of fuel usage. A critical point of the method is determining how the rotation point should move. The strategy we have taken is to compute the acceleration of the rotation point $a_{R}$ using a simple control law

$$
a_{R}=k_{p}\left(r_{u f}-r_{R}\right)-k_{d} v_{R}
$$

where $v_{R}$ is the velocity of the rotation point in the inertial frame. The acceleration of the rotation center is integrated twice to produce the velocity and position at each instant during the retargeting of the constellation.
By so doing, the motion of the rotation center is effectively given second-order dynamics.

With the acceleration, velocity, and position of the rotation center known, the accelerations of the individual spacecraft $a_{i}$ can be determined. It is assumed that the spacecraft rotate as a rigid body about the moving center of rotation according to the trajectory $\theta(t)$. The individual spacecraft accelerations are the basis for calculation of fuel usage rates $\dot{f}_{i}$ which are integrated to determine the available fuel mass $f_{i}$ for each spacecraft: $\dot{f}_{i}=-\gamma M_{i} a_{i}$.

Throughout the duration of the constellation rotation, the available fuel of each spacecraft is monitored. Based on the fuel distribution in the constellation, the point of rotation is moved so that smaller accelerations (and thus less fuel usage) are required of those spacecraft running low on fuel. The final outcome of this procedure is a trajectory for each spacecraft in the constellation that results in fuel usage being as uniform as possible for a specific reorientation of the constellation.

\section{Simulation Results}

This section describes simulation results using the two approaches described in Sections 3 and 4. Simulations were performed in Matlab. For both fixed and dynamic points of rotation, the desired motion of the constellation is obtained from the kinematic equations given in Equation (6). Desired position, velocity and acceleration trajectories for each spacecraft are obtained from the kinematics of the constellation. Each spacecraft tracks its desired trajectory via PD control.

The spacecraft are given the initial positions in the constellation of $r_{1}^{C}=(0,0)^{T}, r_{1}^{C}=(1000,0)^{T}, r_{1}^{C}=$ $(940,342)^{T}, r_{1}^{C}=(940,-342)^{T}$ (units are meters). For fixed point of rotation, $\lambda$ is always set equal to one. Figure 2 shows a bar chart of $f_{i}\left(t_{f}\right)$, the fuel after the constellation undergoes a $\hat{\theta}=90$ degree rotation. Subplots (a), (b), (c), and (d) show $f_{i}\left(t_{f}\right)$ for $\mu=0,1000,3000,10^{5}$ respectively. As can be seen from equation (2), $\mu=0$ corresponds to fuel minimization, whereas $\mu=10^{5}$ corresponds to fuel equalization. Indeed the total fuel usage for $\mu=0,1000,3000,10^{5}$, is $F=9.30,9.98,10.64,11.27$ respectively.

Figure 3 shows the trajectories of the constellation for a dynamic point of rotation. The initial position of the spacecraft are denoted by ' $x$ ' while the final positions are denoted by 'o'. The initial and final position of the point of rotation is depicted as ' $\mathrm{x}$ ' and '*' respectively. The corresponding bar chart of $f_{i}\left(t_{f}\right)$ are given in Figure 4. The total fuel consumed by the constellation in cases (a), (b), (c), and (d) are 10.46, 10.32, 13.27, and 15.61 respectively. Figure 3(a) shows the trajectory of the constellation where the initial rotation point is given by the fixed point algorithm with $\mu=10^{5}$, i.e. $r_{R}=(516.6,0)^{T}$. Note 

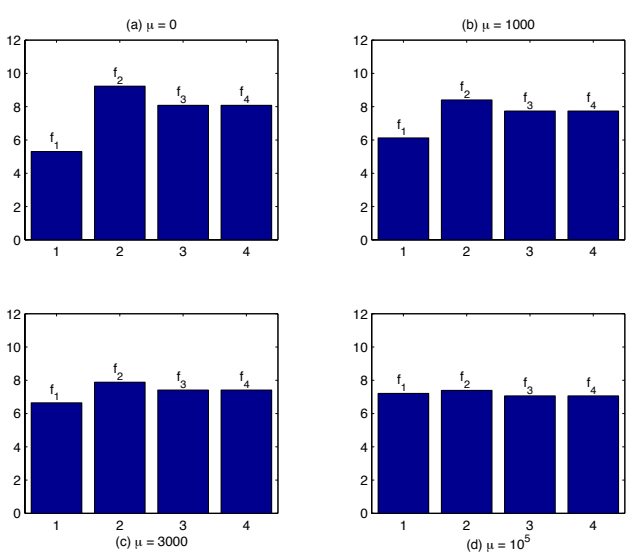

Figure 2: The final fuel distribution for a fixed point of rotation.

that the point of rotation does not stay fixed as might be expected. This is because the fixed point algorithm finds the point that equalizes the fuel over the entire trajectory, whereas the dynamic point algorithm moves the point of rotation to equalize the fuel locally in time. Figure 3(b) shows the trajectories of the constellation where the initial point of rotation is given by the fixed point algorithm when $\mu=0$, i.e. $r_{R}=(859.2,0)^{T}$. Note that the point of rotation dynamically moves toward the center of the constellation. Figure 3(c) shows the trajectories of the constellation when the initial point of rotation is $r_{R}=(516.6,0)^{T}$ but the $3^{\text {rd }}$ spacecraft starts with half as much fuel as the rest of the spacecraft, i.e. $f_{3}(0)=5$. Note that the point of rotation moves toward the $3^{\text {rd }}$ spacecraft to try to equalize the fuel. Figure $3(\mathrm{~d})$ shows the trajectories of the constellation when the initial point of rotation is $r_{R}=(516.6,0)^{T}$ but the $4^{\text {th }}$ spacecraft burns fuel at three times the rate as the other spacecraft, i.e. $\gamma_{4}=3 \gamma_{i}, i=1,2,3$. Note that the point of rotation again moves toward the $4^{\text {th }}$ spacecraft to account for the unequal rate of fuel consumption.

While picking the point of rotation dynamically is more robust, the overall fuel consumption is increased since the accelerations increase for each spacecraft as the point of rotation is moved.

\section{Acknowledgment}

This work was sponsored by the Jet Propulsion Laboratory, California Institute of Technology, under contract with the National Aeronautics and Space Administration.

\section{References}

[1] P. K. C. Wang and F. Y. Hadaegh, "Coordination
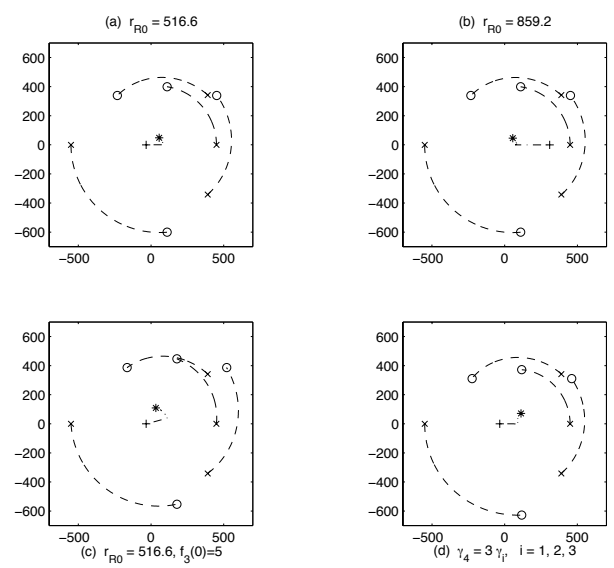

Figure 3: Constellation Trajectories for a dynamic point of rotation.
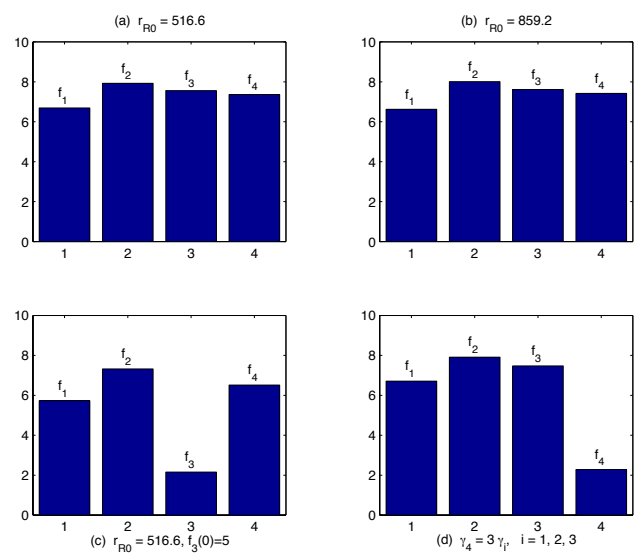

Figure 4: The final fuel distribution for a dynamic point of rotation.

and control of multiple microspacecraft moving in formation," The Journal of the Astronautical Sciences, vol. 44, no. 3, pp. 315-355, 1996.

[2] P. K. C. Wang, F. Y. Hadaegh, and K. Lau, "Synchronized formation rotation and attitude control of multiple free-flying spacecraft," in Proceedings of the AIAA Navigation, Guidance and Control Conference, 1997.

[3] R. Beard, R. Frost, and W. Stirling, "A hierarchical coordination scheme for satellite formation initialization," in AIAA Guidance, Navigation and Control Conference, (Boston, MA), 1998.

[4] T. M. Cover and J. A. Thomas, Elements of Information Theory. Wiley, 1991.

[5] M. Athans and P. Falb, Optimal Control. New York, New York: McGraw-Hill Book Company, 1966. 